\title{
Robust Soft-Decision Interpolation Using Weighted Least Squares
}

\author{
Kwok-Wai Hung, Student Member, IEEE, and Wan-Chi Siu, Fellow, IEEE
}

\begin{abstract}
Soft-decision adaptive interpolation (SAI) provides a powerful framework for image interpolation. The robustness of SAI can be further improved by using weighted least-squares estimation, instead of least-squares estimation in both of the parameter estimation and data estimation steps. To address the mismatch issue of "geometric duality" during parameter estimation, the residuals (prediction errors) are weighted according to the geometric similarity between the pixel of interest and the residuals. The robustness of data estimation can be improved by modeling the weights of residuals with the well-known bilateral filter. Experimental results show that there is a $0.25-\mathrm{dB}$ increase in peak signal-to-noise ratio (PSNR) for a sample set of natural images after the suggested improvements are incorporated into the original SAI. The proposed algorithm produces the highest quality in terms of PSNR and subjective quality among sophisticated algorithms in the literature.
\end{abstract}

Index Terms-Edge-directed interpolation, image interpolation, soft decision, weighted least squares (WLS).

\section{INTRODUCTION}

A N APPROACH using 2-D autoregressive modeling and soft-decision interpolation was proposed by Zhang and $\mathrm{Wu}$ recently [1]. This method is called soft-decision adaptive interpolation (SAI), which is one of the state-of-the-art algorithms in the literature. It provides the highest average peak signal-to-noise ratio (PSNR) (in decidels) among some sophisticated edge-directed algorithms [2]-[4]. Furthermore, some other interpolation and superresolution algorithms were also proposed recently [5]-[7]. In this paper, the SAI method is formulated as the maximum a posterior (MAP) estimation problem, which involves two steps of parameter estimation and data estimation. Similar to new edge directed interpolation (NEDI) [3], SAI relies on "geometric duality" (i.e., the consistency of geometric structure across resolutions) to estimate $H R$ parameters using $L R$ samples. Fig. 1 shows that the LR samples resemble the HR samples due to consistent geometric structure, which is defined according to the image model. However, "geometric duality" is not well satisfied in some cases. Let us use Fig. 2, which shows an HR patch extracted from the

Manuscript received December 02, 2010; revised March 21, 2011 and June 28, 2011; accepted August 24, 2011. Date of publication September 19, 2011; date of current version February 17, 2012. This work was supported in part by the Center for Signal Processing, the Hong Kong Polytechnic University, and the Research Grant Council of the Hong Kong SAR Government under Grant PolyU 5278/08E. The associate editor coordinating the review of this manuscript and approving it for publication was Prof. Xiaolin Wu.

The authors are with the Centre for Signal Processing, Department of Electronic and Information Engineering, The Hong Kong Polytechnic University, Hong Kong (e-mail: enwcsiu@polyu.edu.hk).

Color versions of one or more of the figures in this paper are available online at http://ieeexplore.ieee.org.

Digital Object Identifier 10.1109/TIP.2011.2168416

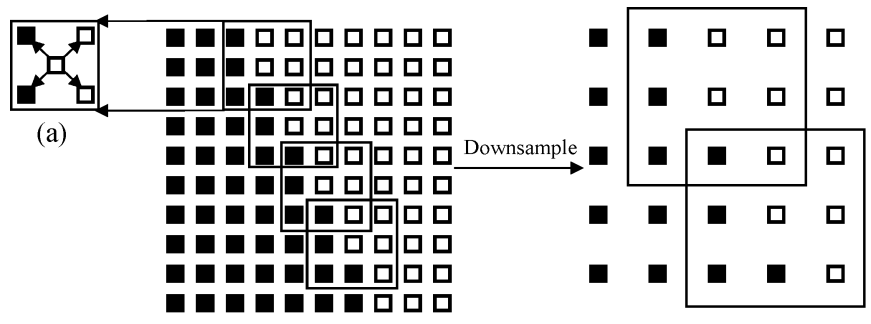

(b)

(c)

Fig. 1. "Geometric duality." (a) HR geometric structure. (b) Repeating in the HR image. (c) Repeating in the downsampled LR image.

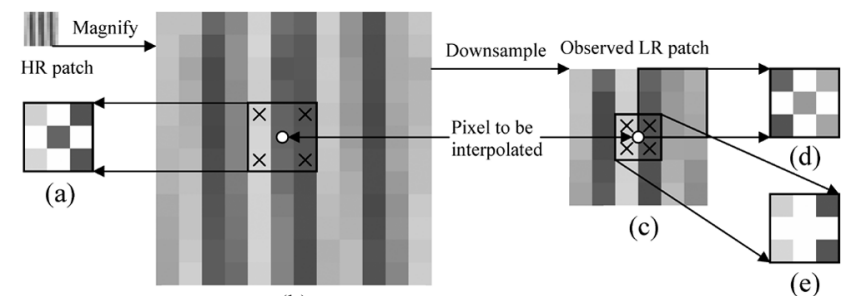

(b)

Fig. 2. Failure of "geometric duality." (a) HR geometric structure. (b) HR patch. (c) Observed LR patch due to a mismatch of "geometric duality." (d) Randomly sampled LR geometric structures. (e) Partial HR geometric structure without the center value.

lighthouse image, to illustrate this situation. Fig. 2(d) shows a randomly sampled geometric structure in the observed LR patch. Most samples in the observed LR patch are irrelevant to the HR geometric structure of interest [ Fig. 2(a)], such that the estimated parameter using the samples in the observed LR patch cannot match well to the HR geometric structure for interpolating the missing pixel, which means a mismatch of "geometric duality." Answers to this problem have not been well addressed in the literature.

SAI makes use of least-squares estimation (for both parameter and data estimation steps), which is equivalent to the maximum likelihood and MAP estimation using the white Gaussian noise assumption in the image model. It is well known that the least-squares estimation is not robust to outliners. During the last decades, the majority of solutions suggested using a robust norm, such as $L_{1}$ norm used by superresolution [8] and adaptive norm used by Huber function [9], instead of $L_{2}$ norm, which results in using least-squares estimation. Weighted least-squares (WLS) estimation is another more intuitive solution, which multiplies a weighting matrix to residuals, making minimum modification to the original solution.

Recently, more NEDI-related algorithms were developed. Xiong et al. [10] proposed a tanner-graph-based interpolation method using NEDI's method for parameter estimation. Zhang et al. [11] suggested using nonlocal means for weighting the 
residuals for NEDI; Liu et al. [12] proposed a regularized local linear regression algorithm, and the samples are weighted by combining nonlocal means and bilateral filter. In an early version of our work [13], we also model the weights of residuals for parameter estimation using the bilateral filter. However, the aforementioned weighting methods lack theoretical analysis, and the patch sizes used do not exactly match the image model. Furthermore, the difference in the patch scales in the HR and LR patches due to downsampling is also ignored.

In this paper, WLS estimation is applied to the SAI method for robustness in both parameter and data estimations. For parameter estimation, the weighting criteria are specifically designed according to the definition of "geometric duality" and the image model for weighting the residuals, as shown in Fig. 2. The criterion called geometric similarity includes two terms: 1) the structural similarity (SS) to measure the difference between the HR structure of interest [ Fig. 2(e)] and LR structures of the residuals [ Fig. 2(d)], and 2) the geometric distance to decay the weights of the residuals according to their spatial distances from the pixel of interest. The proposed weighting criteria are analyzed and can potentially model the probability of "geometric duality," which is the basic assumption of parameter estimation. Thus, the weights of unfavorable residuals (which violate "geometric duality") can be reduced, leading to robust estimation. For data estimation, the weighting criteria are simplified from that in parameter estimation, and it resembles the well-known bilateral filter [14], which has been successfully shown to be a weighting model for parameter estimation in kernel regression [15].

We have implemented the SAI and found that it has a slightly worse PNSR $(0.13 \mathrm{~dB})$ performance compared to the official release from [16]. Nonetheless, it was found that the original SAI method was optimal using the autoregressive model, which utilizes a small local window (12 pixels) for both parameter estimation and data estimation, and interpolates 4 pixels per shift in a raster order. The SAI method using a small local window can yield a low probability of the occurrence of outliners. Little improvement $(0.05 \mathrm{~dB})$ was shown if the proposed WLS estimations are adapted to our implementation of the original formulation of the SAI.

To maximize the performance of the WLS estimation, the SAI is modified by using a larger local window for parameter estimation and a smaller window for data estimation. The large local window can favor the proposed WLS estimation since more useful information can be used while the robustness and accuracy are maintained by using adaptive weights. A smaller local window for data estimation can estimate 9 pixels per window, while only 1 pixel is replaced each time, such that the modified SAI becomes a pixel-based estimation algorithm. Pixel-based data estimation can make the estimation localize to one pixel of interest at one time. Before applying the WLS to the modified SAI (a change in window sizes only), the modified SAI gives similar performance to the original SAI (based on our implementation) in terms of PSNR. Applying the WLS approach to the modified SAI eventually gives a better PSNR $(0.25 \mathrm{~dB}$ on average) than the official release of SAI from [16]. The results agree with the reason using the WLS estimation.

The organization of the rest of this paper is given as follows: Section II describes the formulation of the SAI method using MAP estimation. Section III presents WLS estimation

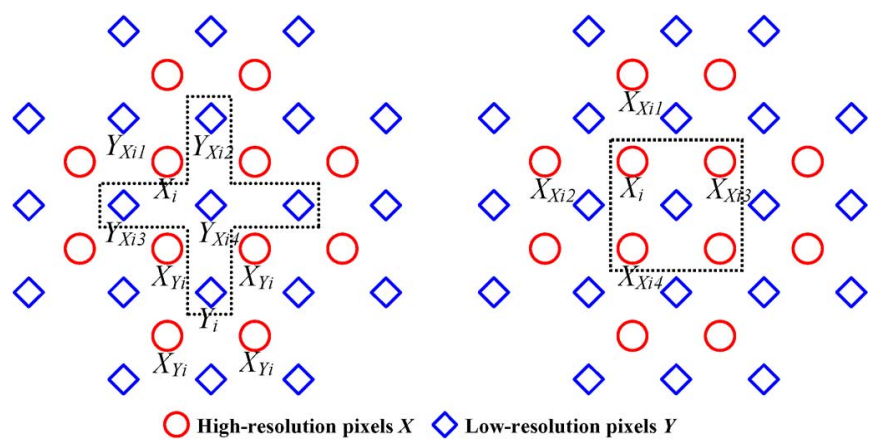

Fig. 3. Graphical illustration of $21 \mathrm{LR}$ and $12 \mathrm{HR}$ pixels within the local window used by SAI. The left figure shows one element of $X_{i} \in \boldsymbol{X}$ and $Y_{i} \in \boldsymbol{Y}$, and their diagonal neighbors $Y_{X i j} \in \boldsymbol{Y}_{\boldsymbol{X}}$ and $X_{Y i j} \in \boldsymbol{X}_{\boldsymbol{Y}}$, where $i$ and $j$ represent the row and column indexes. The right figure shows one element $X_{i} \in \boldsymbol{X}$ and its horizontal-vertical neighbors $X_{X i j} \in \boldsymbol{X}_{\boldsymbol{X}}$.

using geometric similarity and bilateral filter for modeling the weights. Experimental results are shown in Section IV, and a conclusion is given in Section V.

\section{SAI [1]}

Let us formulate the interpolation problem as a MAP estimation. Within a local window as shown in Fig. 3, the observed LR pixels $\boldsymbol{Y}$ in the LR image is used to predict the HR pixels $\boldsymbol{X}$ in the HR image. The LR and HR pixels are represented by columnwise lexicographically ordered matrix notations for convenience. Throughout this paper, the nonbold symbols relating to the matrixes represent their respective elements. The sizes of $\boldsymbol{X}$ and $\boldsymbol{Y}$ in this section (the original SAI) are $12 \times 1$ and $21 \times 1$, respectively. The MAP estimation of $\boldsymbol{X}$ is given by

$$
\underset{\boldsymbol{X}}{\arg \max } p(\boldsymbol{X} / \boldsymbol{Y})=\underset{\boldsymbol{X}}{\arg \max } p(\boldsymbol{Y} / \boldsymbol{X}) p(\boldsymbol{X})
$$

where maximizing the posterior probability is equivalent to maximizing the likelihood multiplied with the prior probability. In SAI, the likelihood is assumed to follow Gaussian distribution with equal variances (unimportant constants are ignored) as follows:

$$
p(\boldsymbol{Y} / \boldsymbol{X})=\exp \left(-\frac{\left\|\boldsymbol{X}-\boldsymbol{Y}_{\boldsymbol{X}} \boldsymbol{A}\right\|_{2}^{2}+\left\|\tilde{\boldsymbol{Y}}-\boldsymbol{X}_{\boldsymbol{Y}} \boldsymbol{A}\right\|_{2}^{2}}{\sigma_{A}}\right)
$$

where $\exp ($.$) means exponential operation, the elements of \boldsymbol{A}$ are the model parameters, $\boldsymbol{Y}_{\boldsymbol{X}}$ are the diagonal neighbors of $\boldsymbol{X}$, and $X_{Y}$ are the diagonal neighbors of $\tilde{Y}$. The sizes of $A, Y_{X}, X_{Y}$, and $\widetilde{Y}$ are $4 \times 1,21 \times 4,5 \times 4$, and $5 \times 1$, respectively. The size of $\widetilde{Y}$ is determined to guarantee that its neighbors $X_{Y}$ are defined within the local window. More specifically, the elements of $\widetilde{\boldsymbol{Y}}$ are the five centermost elements of $\boldsymbol{Y}$ (bounded by dotted cross in Fig. 3). Note that the elements of $\boldsymbol{Y}_{\boldsymbol{X}}$ and $\widetilde{\boldsymbol{Y}}$ are subsets of the elements of $\boldsymbol{Y}$. On the contrary, all the elements of $\boldsymbol{Y}$ have counterparts in $\boldsymbol{Y}_{\boldsymbol{X}}$ and/or $\widetilde{\boldsymbol{Y}}$. Similarly, the elements of $X_{Y}$ are subsets of the elements of $X$, and all the elements of $\boldsymbol{X}$ have counterparts in $\boldsymbol{X}_{\boldsymbol{Y}}$ and/or $\boldsymbol{X}$. Hence, all LR and HR pixels in $\boldsymbol{Y}$ and $\boldsymbol{X}$ are defined in the likelihood. Examples of these symbols are shown in Fig. 3. The Gaussian distribution of the likelihood is equivalent to the zero-mean white Gaussian noise assumption in the image models, $X=Y_{X} A+n$ and $\widetilde{Y}=X_{Y} A+n$, where $n$ is the noise. Similarly, assume that the 


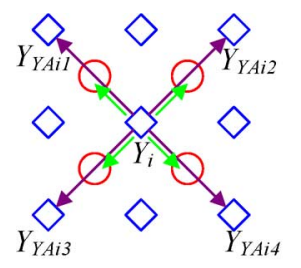

(a)

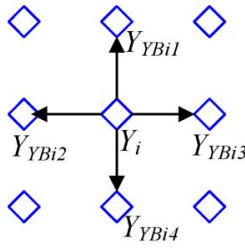

(b)

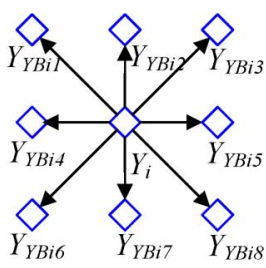

(c) $\bigcirc$ High-resolution pixels $X \diamond$ Low-resolution pixels $Y$

$\longrightarrow$ Estimated LR parameter $\longrightarrow$ HR parameter

Fig. 4. (a) Graphical illustration of the spatial difference between the estimated LR parameter and the HR parameter. (b) Original horizontal-vertical correlations. (c) Proposed all-rounded correlations.

prior probability follows Gaussian distribution with a different variance, as follows:

$$
p(\boldsymbol{X})=\exp \left(-\frac{\left\|\tilde{\boldsymbol{X}}-\boldsymbol{X}_{\boldsymbol{X}} \boldsymbol{B}\right\|_{2}^{2}}{\sigma_{B}}\right)
$$

where the elements of $\boldsymbol{B}$ are the model parameters, and $\boldsymbol{X}_{\boldsymbol{X}}$ are the horizontal-vertical neighbors of $\widetilde{\boldsymbol{X}}$. Similarly, the size of $\widetilde{\boldsymbol{X}}$ is determined to guarantee that its neighbors $\boldsymbol{X}_{\boldsymbol{X}}$ are defined within the local window. More specifically, $\widetilde{X}$ is a $4 \times 1$ vector representing the centermost four elements of $\boldsymbol{X}$ (bounded by dotted square in Fig. 3). Note that the elements of $\widetilde{\boldsymbol{X}}$ and $\boldsymbol{X}_{\boldsymbol{X}}$ are subsets of the elements of $\boldsymbol{X}$. On the contrary, all the elements of $\boldsymbol{X}$ have counterparts in $\widetilde{\boldsymbol{X}}$ and/or $\boldsymbol{X}_{\boldsymbol{X}}$ and/or $\boldsymbol{X}_{\boldsymbol{Y}}$. Hence, all the HR pixels in $\boldsymbol{X}$ are defined in the prior probability. One sample of these symbols is shown in Fig. 3 (right). The model parameters are estimated using the least-squares estimation approach as follows:

$$
\begin{gathered}
\underset{\boldsymbol{A}}{\arg \min }\left\|\boldsymbol{Y}-\boldsymbol{Y}_{\boldsymbol{Y} \boldsymbol{A}} \boldsymbol{A}\right\|_{2}^{2} \\
\underset{\boldsymbol{B}}{\arg \min }\left\|\boldsymbol{Y}-\boldsymbol{Y}_{\boldsymbol{Y} \boldsymbol{B}} \boldsymbol{B}\right\|_{2}^{2}
\end{gathered}
$$

where $\boldsymbol{Y}_{\boldsymbol{Y} \boldsymbol{A}}$ are the diagonal neighbors of $\boldsymbol{Y}$, and $\boldsymbol{Y}_{\boldsymbol{Y} B}$ are the horizontal-vertical neighbors of $\boldsymbol{Y}$, as shown in Fig. 4(a) and (b). Note that the size of $\boldsymbol{Y}, \boldsymbol{Y}_{\boldsymbol{Y}}$, and $\boldsymbol{Y}_{\boldsymbol{Y} B}$ in (4) and (5) are $21 \times 1,21 \times 4$, and $21 \times 4$, respectively. Parameters estimated using LR pixels refer to an approximation of the HR parameters by using the assumption of "geometric duality." The situation is shown in Fig. 4(a). In this figure, the parameters of the HR model relate the diagonal pixels with the center pixel by one half of the distance of the estimated LR parameters. Although the least-squares estimations in (4) and (5) are not robust to outliners, SAI uses a small window size, which has a low possibility of including outliners in general cases. The MAP estimation in (1) can be simplified by taking logarithm as the following:

$$
\begin{aligned}
& \underset{\boldsymbol{X}}{\arg \max } p(\boldsymbol{X} / \boldsymbol{Y}) \\
& =\underset{\boldsymbol{X}}{\arg \max } \log [p(\boldsymbol{Y} / \boldsymbol{X}) p(\boldsymbol{X})] \\
& \quad=\underset{\boldsymbol{X}}{\arg \min } \frac{\left\|\boldsymbol{X}-\boldsymbol{Y}_{\boldsymbol{X}} \boldsymbol{A}\right\|_{2}^{2}}{\sigma_{A}}+\frac{\left\|\widetilde{\boldsymbol{Y}}-\boldsymbol{X}_{\boldsymbol{Y}} \boldsymbol{A}\right\|_{2}^{2}}{\sigma_{A}}+\frac{\left\|\widetilde{\boldsymbol{X}}-\boldsymbol{X}_{\boldsymbol{X}} \boldsymbol{B}\right\|_{2}^{2}}{\sigma_{B}} \\
& \quad \underset{\boldsymbol{X}}{\arg \min }\left\|\boldsymbol{X}-\boldsymbol{Y}_{\boldsymbol{X}} \boldsymbol{A}\right\|_{2}^{2}+\left\|\widetilde{\boldsymbol{Y}}-\boldsymbol{X}_{\boldsymbol{Y}} \boldsymbol{A}\right\|_{2}^{2}+\lambda\left\|\widetilde{\boldsymbol{X}}-\boldsymbol{X}_{\boldsymbol{X}} \boldsymbol{B}\right\|_{2}^{2}
\end{aligned}
$$

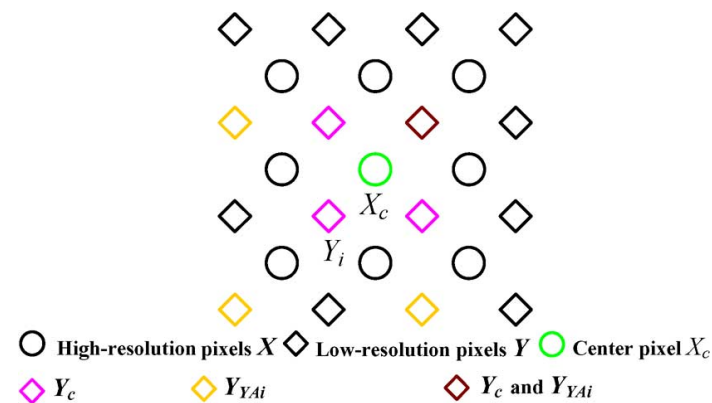

Fig. 5. Center part of the $8 \times 8$ window used for parameter estimation: center pixel and its diagonal neighbors $Y_{c}$ (which represent the HR geometric structure), and one LR sample $Y_{i}$ and its diagonal neighbors $Y_{Y A i}$ (which represent the LR geometric structure).

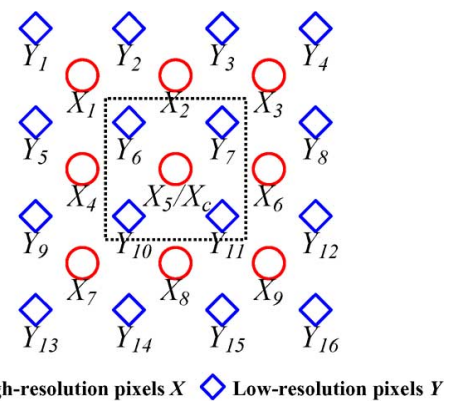

Fig. 6. Local window used for data estimation for the proposed method.

where $\lambda$ is the regularization factor that was recommended to be 0.5 [1]. Solving the least-squares estimation of the parameter estimation in (4) and (5) within a local window yields an autoregressive model with white Gaussian noise assumption. Estimation of the high-resolution pixels using (6) is called SAI, and (6) can be then solved accordingly [1].

\section{WLS ESTIMATION USING GEOMETRIC SIMILARITY AND BILATERAL FILTER}

WLS estimation is a powerful and intuitive solution to reduce the influence of outliners and maintain robustness. The mismatch issue of "geometric duality" is a kind of outliners. For example, the estimated LR parameters do not approximate the HR parameters when the geometric structures change across resolutions in Fig. 4(a). In Section III-A, we propose a novel weighting method during the parameter estimation to better approximate the ground true HR parameters. In Section III-B, we introduce the bilateral filter as the weighting method during the data estimation. Note that, to achieve the maximum performance of WLS, the local window for parameter estimation in (4) and (5) is enlarged to $8 \times 8$ (in terms of LR pixels), and the local window for data estimation in (6) is reduced to $3 \times 3$ (in terms of $\mathrm{HR}$ pixels), as explained in the introduction section (Figs. 5 and 6).

\section{A. Parameter Estimation}

Due to the enlargement of the local window to $8 \times 8$ (in terms of LR pixels), the size of $\boldsymbol{Y}$ in this section is $64 \times 1$. The ordinary least-squares estimation in (4) and (5) is equivalent to maximum-likelihood estimation with white Gaussian noise assumption. The Gaussian distribution is used since it gives us the least-squares solution, which minimizes the least-squared error. 
Let us relax the white noise assumption and consider the covariance matrix $\boldsymbol{V}$, which fully characterizes the multivariate Gaussian distribution as follows:

$$
\underset{\boldsymbol{A}}{\arg \min }\left(\boldsymbol{Y}-\boldsymbol{Y}_{\boldsymbol{Y} \boldsymbol{A}} \boldsymbol{A}\right)^{T} \boldsymbol{V}^{-1}\left(\boldsymbol{Y}-\boldsymbol{Y}_{\boldsymbol{Y} \boldsymbol{A}} \boldsymbol{A}\right)
$$

and $V_{i j} \in \boldsymbol{V}$, where $V_{i j}=E\left[\left(Y_{i}-\boldsymbol{Y}_{\boldsymbol{Y} \boldsymbol{A} i} \boldsymbol{A}\right)\left(Y_{j}-\boldsymbol{Y}_{\boldsymbol{Y} \boldsymbol{A} \boldsymbol{j}} \boldsymbol{A}\right)\right]$. To analyze the covariance matrix, we can use the sample covariance matrix obtained by applying the following cost function (derived from MAP estimation) to obtain several sets of ground true parameters, which perfectly reconstruct the HR center pixel $\left(X_{c}\right)$ [the fifth element of $\boldsymbol{X}$ in (16)] from its four LR diagonal pixels $\left(\boldsymbol{Y}_{\boldsymbol{c}}\right)$ (see Fig. 5)

$$
\underset{\boldsymbol{A}}{\arg \min }\left\{\left(X_{c}-\boldsymbol{Y}_{\boldsymbol{c}} \boldsymbol{A}\right)^{2}+\alpha[\boldsymbol{I} \boldsymbol{A}-1]^{2}\right\}
$$

where $\boldsymbol{I}=\left[\begin{array}{llll}1 & 1 & 1 & 1\end{array}\right]$, and $\alpha$ is a regularization factor to regularize the sum of elements of $\boldsymbol{A}$ (i.e., $\boldsymbol{I} \boldsymbol{A}$ ) to 1 for filter stability; such stability is naturally inherited when samples in the smooth region are used. (The samples in the smooth regions are regularizers, ${ }^{1}$ which regularize the sum of parameters to 1 .) For generalization, we incorporate such regularization into our analysis of covariance. By solving (8) using the gradient decent, we obtained the following iteration equation:

$$
\boldsymbol{A}^{n+1}=\boldsymbol{A}^{n}+\sigma\left\{\boldsymbol{Y}_{\boldsymbol{c}}^{T}\left(X_{c}-\boldsymbol{Y}_{\boldsymbol{c}} \boldsymbol{A}^{n}\right)-\alpha \boldsymbol{I}^{T}\left[\boldsymbol{I} \boldsymbol{A}^{n}-1\right]\right\}
$$

where $\sigma$ should be a sufficiently small value that indicates the step size to ensure stable convergence, and the absolute residual $\left|X_{c}-Y_{c} A\right|$ is less than 0.5. Each set of parameters generates 64 residuals $\left(Y_{i}-\boldsymbol{Y}_{\boldsymbol{Y A i}} \boldsymbol{A}\right)$ in the local $8 \times 8$ window (Fig. 5). We sampled uniformly the HR Lena image to obtain about 8000 observations of $X_{c}$ (around the edges) and their corresponding parameters. Since there are infinite sets of ground true parameters that satisfy $\left|X_{c}-\boldsymbol{Y}_{\boldsymbol{c}} \boldsymbol{A}\right|<0.5$, and can be converged by making use of (9), we choose three sets of representative pa-

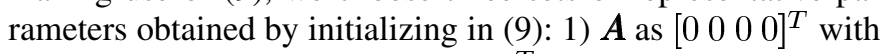

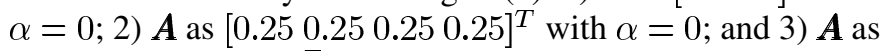
$\left[\begin{array}{llll}0.25 & 0.25 & 0.25 & 0.25\end{array}\right]^{T}$ with $\alpha=10$, which represent the three general settings of parameters.

Let us classify the $8000 \times 64$ observations of residuals and calculate the sample covariance for each class, such that we can analyze the feasibility of estimating the covariance by the proposed criterion of classification. Our proposed method that addresses the "geometric duality" (Fig. 2) includes two criteria. The first criterion is $S S$, i.e., $\left\|\boldsymbol{Y}_{\boldsymbol{c}}-\boldsymbol{Y}_{\boldsymbol{Y} \boldsymbol{A}}\right\|_{p}$, where $\boldsymbol{Y}_{\boldsymbol{c}}$ is defined earlier as the HR geometric structure of interest and $\boldsymbol{Y}_{\boldsymbol{Y A}}$ 's are the LR geometric structures (LR samples). The second criterion is geometric distance, i.e., $\left\|\boldsymbol{Z}_{\boldsymbol{c}}-\boldsymbol{Z}_{\boldsymbol{Y}}\right\|_{p}$, where $\boldsymbol{Z}_{\boldsymbol{c}}$ and $\boldsymbol{Z}_{\boldsymbol{Y}}$ are the $2 \times 1$ vectors that represent the 2-D coordinates of the center pixel (to be interpolated) and LR samples $Y$. The norm was evaluated for $p=1$ and $p=2$. Fig. 5 shows a graphical illustration of one of the samples of the SS, whereas the geometric distance can be understood without graphical illustration.

Due to the square local window and the symmetric property of the covariance matrix, we only calculate the sample covariance of four rows (i.e., $V_{0 j}, V_{9 j}, V_{18 j}$, and $V_{27 j}$ ) and the diag-

\footnotetext{
${ }^{1}$ During the estimation of parameters, the cost function using LR samples in the smooth region is minimized for any parameter $\boldsymbol{A}$, which has a sum of elements equal to 1, i.e., $\boldsymbol{A X}-y=0$ for any $\Sigma A_{i}=1$ when $X_{i}=y$ due to constant intensity in smooth area.
}
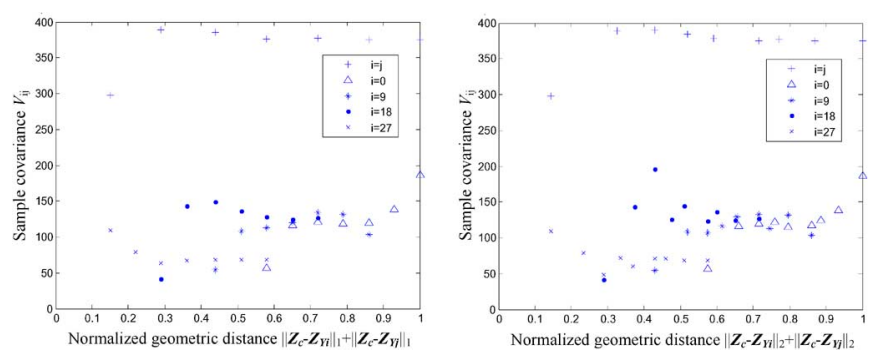

Fig. 7. Plot of sample covariance against the proposed geometric distance criterion.
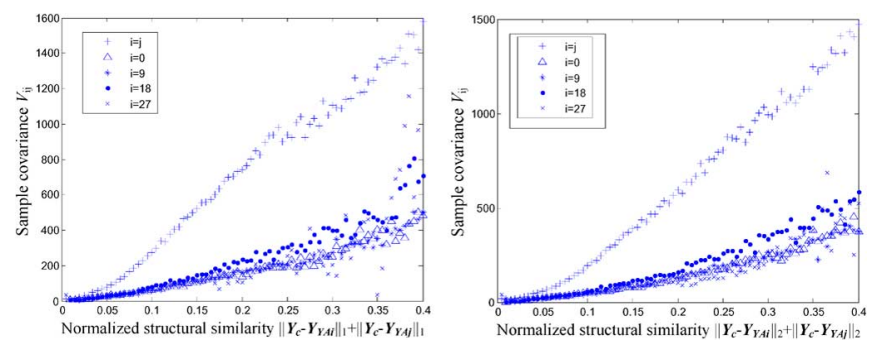

Fig. 8. Plot of sample covariance against the proposed SS criterion.

onal positions (i.e., $V_{i j}$ for $i=j$ ) to account for all positions in the covariance matrix. Our experiments show that the three sets of ground true parameters show very similar characteristics using the proposed criteria; hence, only the figures with the first set of parameters are shown. Figs. 7 and 8 show some plots of sample covariances against the two criteria. Fig. 8 shows that the classified covariances increase exponentially with the $S S$. The row covariances that can represent the nondiagonal covariances follow approximately an exponential distribution with the values of the variances three times of that of the diagonal covariances. The geometric distance is not a good classifier due to the combined effect of outliner and smooth area. Let us refer to Fig. 1 again; the edge only accounts for a small portion in a local window, such that a larger geometric distance often leads to a higher possibility of smooth area and outliners, which compensate each other the results in similar covariances regardless of the geometric distance. Such an effect cannot be revealed by evaluating the covariances; however, the final PSNR values show slight improvements by incorporating the geometric distance criterion (to reduce the effect of outliners since the benefit bought by smooth area is the regularization of parameters), and the proposed function for modeling the covariance is given as follows:

$$
\begin{array}{r}
V_{i j}=\exp \left\{\left(\left\|\boldsymbol{Y}_{\boldsymbol{c}}-\boldsymbol{Y}_{\boldsymbol{Y} \boldsymbol{A} \boldsymbol{i}}\right\|_{p}+\left\|\boldsymbol{Y}_{\boldsymbol{c}}-\boldsymbol{Y}_{\boldsymbol{Y} \boldsymbol{A} \boldsymbol{j}}\right\|_{p}\right) / \sigma_{1 i j}\right\} \\
\cdot \exp \left\{\left(\left\|\boldsymbol{Z}_{\boldsymbol{c}}-\boldsymbol{Z}_{\boldsymbol{Y} \boldsymbol{i}}\right\|_{p}+\left\|\boldsymbol{Z}_{\boldsymbol{c}}-\boldsymbol{Z}_{\boldsymbol{Y} \boldsymbol{j}}\right\|_{p}\right) / \sigma_{2}\right\}
\end{array}
$$

where $\sigma_{1 i j(i=j)}=0.33 \sigma_{1 i j(i \neq j)}$. We evaluate the performance of this definition of covariance matrix using (7) and the proposed overall algorithm. It was found that the full covariance matrix definition with nondiagonal covariance produces 1 -dB-worse performance in the final result. It is due to the fact that our model in (10) does not have negative value of covariance, and in fact, the nondiagonal covariance often has negative value (depending on the definition of $E()$.$) , which does not exist in the sample$ covariances due to the averaging effect of samples. (If less samples are used, instability is shown in Fig. 8 for large values in the 

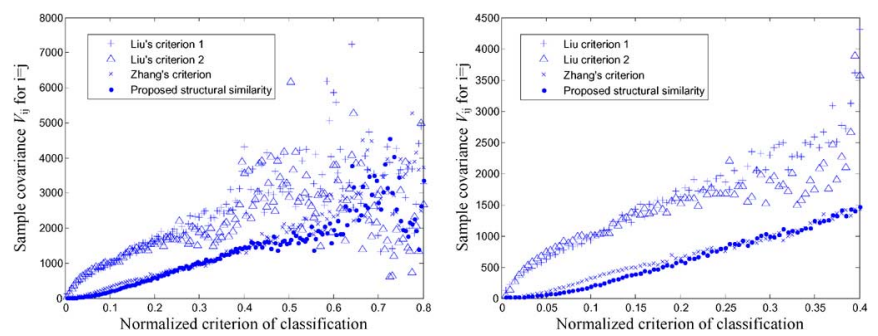

Fig. 9. Plot of sample covariance against different criteria of classification.

$x$-axis.) Hence, the assumption of all positive values of covariances in our model produces unacceptable results. As a result, the nondiagonal covariance is often assumed to be zero, such that the covariance matrix becomes a diagonal matrix, which resembles the inverse of the weighting matrix used in the WLS method [11]-[13]. Let us replace the covariance matrix in (7) by $\boldsymbol{V}^{-1}=\boldsymbol{W}$ (where $\boldsymbol{W}$ is a diagonal matrix for weighting) to obtain the WLS as follows:

$$
\underset{\boldsymbol{A}}{\arg \min }\left\|\boldsymbol{W}^{1 / 2}\left(\boldsymbol{Y}-\boldsymbol{Y}_{\boldsymbol{Y} \boldsymbol{A}} \boldsymbol{A}\right)\right\|_{2}^{2}
$$

where the elements of $W$ are the weighting parameters for the residuals in $\left(\boldsymbol{Y}-\boldsymbol{Y}_{\boldsymbol{Y} A} \boldsymbol{A}\right)$. From (10), we propose the following weighting matrix for WLS estimation:

$$
\begin{aligned}
W_{i}=\underset{i=j}{V_{i j}^{-1}}=\exp \left(-2\left\|\boldsymbol{Y}_{\boldsymbol{c}}-\boldsymbol{Y}_{\boldsymbol{Y} \boldsymbol{A} \boldsymbol{i}}\right\|_{p} / \sigma_{1}\right) \\
\cdot \exp \left(-2\left\|\boldsymbol{Z}_{\boldsymbol{c}}-\boldsymbol{Z}_{\boldsymbol{Y} \boldsymbol{i}}\right\|_{p} / \sigma_{2}\right)
\end{aligned}
$$

which appears to be similar to the weighing methods in [11] and [12]. To analyze the difference between methods, we plot the three criteria (i.e., radiometric distance of bilateral filter using squared $L_{2}$ norm, patch radiometric distance of nonlocal means using squared $L_{2}$ norm, and using $L_{2}$ norm) used in the methods in [11] and [12] according to the previously described procedure in Fig. 9, which shows that the proposed SS provides the best exponential shape. However, since there are infinite sets of parameters that satisfy (8), we also rely on PSNR evaluation using these two methods [11], [12] in our overall algorithm. The results show a drop in PSNR of 0.06 and $0.09 \mathrm{~dB}$ in the Lena image with variances empirically optimized. These methods use the patch size that does not match with the image model used in NEDI [see Fig. 1(a)], and the patch scale does not involve the scale change due to downsampling of the image [see Fig. 2(d) and (e)].

Occurrence of Outliners: Outliners during the parameter estimation are defined statistically as large residuals. Due to the fact that the expected squared residual is a variance that can be modeled by our proposed SS, the outlying residuals can also be approximately modeled by SS. A larger SS means a higher possibility of an outlier. To verify that the least squares estimation of parameters using a small local window involves significant outliners, we have calculated the average SS of the centermost 16 residuals in the local window for edge pixels and plotted the probability of the average SS in Fig. 10. This figure shows that the probability is large (e.g., 0.003) for a large value of average SS in the Lighthouse image. This verifies the occurrence of outliners in small local windows of a natural image. Moreover, we have also calculated the edge mean squared errors in Fig. 10.
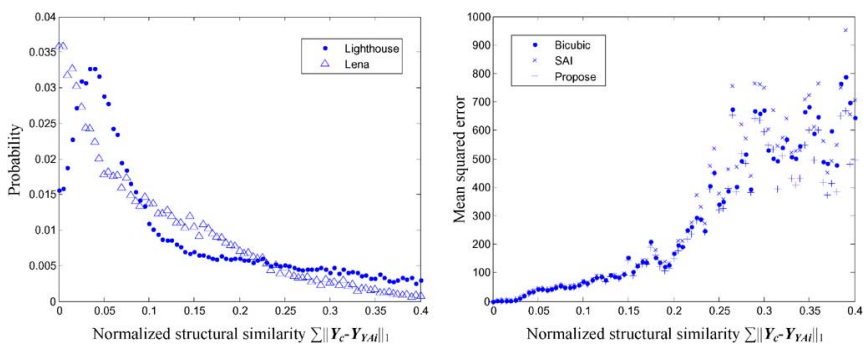

Fig. 10. Plot of probability and mean squared error against the proposed SS criterion.

The result shows that, for a large SS (approximately the outliners), the proposed algorithm provides obviously lower mean squared errors. This further verifies the benefits of WLS for SAI in case of a large SS (approximately the outliners).

WLS Estimation: $L_{1}$ or $L_{2}$ norm $(p=1,2)$ can be used in (12), which produces very similar PSNR when the variances are empirically optimized. Variances $\sigma_{1}$ and $\sigma_{2}$ in (12) are identified empirically (set to 625 and 4 when $p=1$ and $p=2$ for the two terms). By slightly varying these parameters, it hardly changes the PSNR values of the final interpolated image. Hence, the closed-form solution of (11) is given by

$$
\boldsymbol{A}=\left(\boldsymbol{Y}_{Y A}^{T} \boldsymbol{W} \boldsymbol{Y}_{\boldsymbol{Y} \boldsymbol{A}}\right)^{-1} \boldsymbol{Y}_{\boldsymbol{Y} \boldsymbol{A}}^{T} \boldsymbol{W} \boldsymbol{Y} .
$$

Although the estimation of parameter $\boldsymbol{B}$ in (5) does not involve the mismatch issue of "geometric duality" due to the same resolution of the geometric structures in (5) and (6) [see the right-hand side of Figs. 3 and 4(b)], weighting the residuals using $W$ can yield a slightly better result $(0.03 \mathrm{~dB})$ due to the modeling of geometric distance. Due to the relative insignificance of parameter $\boldsymbol{B}$, different modeling methods $(\boldsymbol{W})$ for parameter $B$ show very limited improvement. The closed-form solution of $\boldsymbol{B}$ is given by

$$
\boldsymbol{B}=\left(\boldsymbol{Y}_{Y B}^{T} W \boldsymbol{Y}_{\boldsymbol{Y} \boldsymbol{B}}\right)^{-1} \boldsymbol{Y}_{\boldsymbol{Y} B}^{T} W \boldsymbol{Y}
$$

where $B$ in this paper is modified from horizontal-vertical correlation to all-rounded correlation [as shown in Fig. 4(b) and (c)], which can give a slightly better result $(\sim 0.02 \mathrm{~dB})$. Different from the suggested value of the regularization factor, the ratio of the sum of squared residuals is proposed to define $\lambda$ in (16) as follows:

$$
\lambda=0.5 \frac{\left\|Y-Y_{Y A} A\right\|_{2}^{2}}{\left\|Y-Y_{Y B} B\right\|_{2}^{2}}
$$

which can make the regularization factor adaptive to the estimation accuracy of the two parameters and give a slightly better PSNR $(0.01 \mathrm{~dB})$ on average.

\section{B. Data Estimation}

The local window for data estimation has a size of $3 \times 3$ (in terms of HR pixels), as shown in Fig. 6. The sizes of $\boldsymbol{X}$ and $\boldsymbol{Y}$ in this section are $9 \times 1$ and $16 \times 1$, respectively. Similar to the parameter estimation, we can analyze the sample covariance matrix by substituting the estimated parameters and ground true $X$ into (16). Since the data and parameter estimation use the same image model but different scales, it is anticipated that a similar criterion of classification can be applied. From (10), we modify 

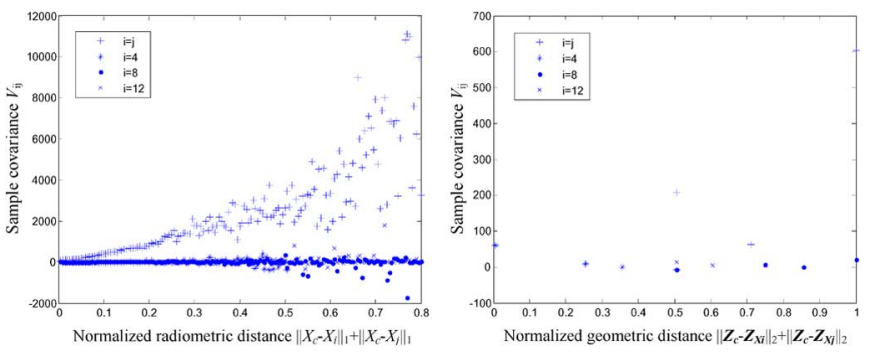

Fig. 11. Plot of sample covariance for data estimation against the proposed criteria.

the SS to involve no scale change and to make it pixel based due to the fact that the patch involves many unknown HR pixels (see Fig. 6) and the resultant criteria resemble the bilateral filter. By following a similar procedure in the parameter estimation, we have plotted the sample covariance classified by the two criteria of the bilateral filter in Fig. 11. The major difference between data and parameter estimation is that the residuals are not correlated since most row covariances (that can represent the off-diagonal covariances) are zero, such that the diagonal definition of covariance matrix should be used. Hence, the data estimation in (6) is modified to the WLS formulation by incorporating the weighting parameters $\boldsymbol{W}_{\boldsymbol{i}}$ as follows:

$$
\begin{array}{r}
\underset{\boldsymbol{X}}{\arg \min }\left\|\boldsymbol{W}_{\mathbf{1}}^{1 / 2}\left(\boldsymbol{X}-\boldsymbol{Y}_{\boldsymbol{X}} \boldsymbol{A}\right)\right\|_{2}^{2}+\left\|\boldsymbol{W}_{\mathbf{2}}^{1 / 2}\left(\tilde{\boldsymbol{Y}}-\boldsymbol{X}_{\boldsymbol{Y}} \boldsymbol{A}\right)\right\|_{2}^{2} \\
+\lambda\left\|\boldsymbol{W}_{\mathbf{3}}^{1 / 2}\left(\tilde{\boldsymbol{X}}-\boldsymbol{X}_{\boldsymbol{X}} \boldsymbol{B}\right)\right\|_{2}^{2}
\end{array}
$$

where $W_{\boldsymbol{i}}$ contains the weighting parameters for residuals in $\left(\boldsymbol{X}-\boldsymbol{Y}_{\boldsymbol{X}} \boldsymbol{A}\right),\left(\tilde{\boldsymbol{Y}}-\boldsymbol{X}_{\boldsymbol{Y}} \boldsymbol{A}\right)$, and $\left(\widetilde{\boldsymbol{X}}-\boldsymbol{X}_{\boldsymbol{X}} \boldsymbol{B}\right)$, respectively. Note that the sizes of $\widetilde{\boldsymbol{Y}}$ and $\widetilde{\boldsymbol{X}}$ are determined in such a way to guarantee that their neighbors $\left(\boldsymbol{X}_{\boldsymbol{Y}}\right.$ and $\left.\boldsymbol{X}_{\boldsymbol{X}}\right)$ would be defined within the local window in Fig. 6. For example, the size of $\widetilde{Y}$ in $\left(\widetilde{\boldsymbol{Y}}-\boldsymbol{X}_{\boldsymbol{Y}} \boldsymbol{A}\right)$ is $4 \times 1$, and the size of $\widetilde{\boldsymbol{X}}$ in $\left(\widetilde{\boldsymbol{X}}-\boldsymbol{X}_{\boldsymbol{X}} \boldsymbol{B}\right)$ is $1 \times 1$. More specifically, $\widetilde{X}\left(=X_{c}\right)$ is a $1 \times 1$ vector representing the centermost element of $\boldsymbol{X}$, and $\tilde{\boldsymbol{Y}}$ is a $4 \times 1$ vector representing the centermost four elements of $\boldsymbol{Y}$ (bounded by dotted square in Fig. 5). All the elements of $\boldsymbol{X}$ have counterparts in $\widetilde{X}$ and/or $\boldsymbol{X}_{\boldsymbol{X}}$ and/or $\boldsymbol{X}_{\boldsymbol{Y}}$. All the elements of $\boldsymbol{Y}$ have counterparts in $\boldsymbol{Y}_{\boldsymbol{X}}$ and/or $\tilde{Y}$. Hence, all the pixels in $X$ and $Y$ are defined in the posterior. We use $\boldsymbol{W}_{\mathbf{1}}$ in the term $\left\|\boldsymbol{W}_{\mathbf{1}}^{1 / 2}\left(\boldsymbol{X}-\boldsymbol{Y}_{\boldsymbol{X}} \boldsymbol{A}\right)\right\|_{2}^{2}$ [ (16)] as an example ( $\boldsymbol{W}_{\boldsymbol{i}}$ in other terms can be derived accordingly) to show the definition of bilateral filter as follows:

$$
W_{1 i}=\exp \left(-\left\|X_{c}-X_{i}\right\|_{1} / 125\right) \cdot \exp \left(-\left\|\boldsymbol{Z}_{\boldsymbol{c}}-\boldsymbol{Z}_{\boldsymbol{X} \boldsymbol{i}}\right\|_{2} / 2\right)
$$

where $\boldsymbol{W}_{1 i} \in \boldsymbol{W}_{\mathbf{1}},\left\|X_{c}-X_{i}\right\|_{1}$ is the radiometric distance between the center pixel $X_{c}$ (approximated by bilinear interpolation for calculating the radiometric distance) and $X_{i}$, and $\left\|Z_{\boldsymbol{c}}-\boldsymbol{Z}_{\boldsymbol{X} \boldsymbol{i}}\right\|_{2}$ is the geometric distance using $L_{2}$ norm between the center pixel and $X_{i}$. Note that each element in $X$ corresponds to one element of $\boldsymbol{W}_{\mathbf{1}}$ in (17). The variances, i.e., 125 and 2, were found empirically, and mildly varying these two parameters hardly changes the PSNR values of the final interpolated image. Setting 125 to $[100,150]$ and 2 to $[1.75,2]$ gives similar results, which gives the highest PSNR values on average. Equation (16) can be reformulated into a more compact representation (similar to the approach in SAI) as follows:

$$
\underset{\boldsymbol{X}}{\arg \min }(\boldsymbol{C X}-\boldsymbol{D} \boldsymbol{Y})^{T} \boldsymbol{W}(\boldsymbol{C} \boldsymbol{X}-\boldsymbol{D} \boldsymbol{Y})
$$

where $\boldsymbol{W}$ is a $14 \times 14$ diagonal matrix [the combination of the three $\boldsymbol{W}_{\boldsymbol{i}}$ in (16)], and $\boldsymbol{D}$ is a $14 \times 16$ matrix defined as (19), shown at the bottom of the page, and $A_{i} \in \boldsymbol{A}$. $\boldsymbol{C}$ is a $14 \times 9$ matrix, and $\boldsymbol{C}=\left[\boldsymbol{I}_{9 \times 9} \boldsymbol{C}_{\mathbf{1}}\right]^{T} . \boldsymbol{I}_{9 \times 9}$ is a $9 \times 9$ identity matrix, and the definition of $\boldsymbol{C}_{\mathbf{1}}$ is given by

$C_{1}$

$=\left[\begin{array}{ccccccccc}A_{1} & A_{2} & 0 & A_{3} & A_{4} & 0 & 0 & 0 & 0 \\ 0 & A_{1} & A_{2} & 0 & A_{3} & A_{4} & 0 & 0 & 0 \\ 0 & 0 & 0 & A_{1} & A_{2} & 0 & A_{3} & A_{4} & 0 \\ 0 & 0 & 0 & 0 & A_{1} & A_{2} & 0 & A_{3} & A_{4} \\ \lambda B_{1} & \lambda B_{2} & \lambda B_{3} & \lambda B_{4} & -\lambda & \lambda B_{5} & \lambda B_{6} & \lambda B_{7} & \lambda B_{8}\end{array}\right]$

and $B_{i} \in \boldsymbol{B}$. Since (18) is a convex optimization problem, its closed-form solution can be derived by putting the derivative into zero and is given by

$$
\boldsymbol{X}=\left(\boldsymbol{C}^{T} \boldsymbol{W} \boldsymbol{C}\right)^{-1} \boldsymbol{C}^{T} \boldsymbol{W} \boldsymbol{D Y}
$$

$$
\boldsymbol{D}=\left[\begin{array}{cccccccccccccccc}
A_{1} & A_{2} & 0 & 0 & A_{3} & A_{4} & 0 & 0 & 0 & 0 & 0 & 0 & 0 & 0 & 0 & 0 \\
0 & A_{1} & A_{2} & 0 & 0 & A_{3} & A_{4} & 0 & 0 & 0 & 0 & 0 & 0 & 0 & 0 & 0 \\
0 & 0 & A_{1} & A_{2} & 0 & 0 & A_{3} & A_{4} & 0 & 0 & 0 & 0 & 0 & 0 & 0 & 0 \\
0 & 0 & 0 & 0 & A_{1} & A_{2} & 0 & 0 & A_{3} & A_{4} & 0 & 0 & 0 & 0 & 0 & 0 \\
0 & 0 & 0 & 0 & 0 & A_{1} & A_{2} & 0 & 0 & A_{3} & A_{4} & 0 & 0 & 0 & 0 & 0 \\
0 & 0 & 0 & 0 & 0 & 0 & A_{1} & A_{2} & 0 & 0 & A_{3} & A_{4} & 0 & 0 & 0 & 0 \\
0 & 0 & 0 & 0 & 0 & 0 & 0 & 0 & A_{1} & A_{2} & 0 & 0 & A_{3} & A_{4} & 0 & 0 \\
0 & 0 & 0 & 0 & 0 & 0 & 0 & 0 & 0 & A_{1} & A_{2} & 0 & 0 & A_{3} & A_{4} & 0 \\
0 & 0 & 0 & 0 & 0 & 0 & 0 & 0 & 0 & 0 & A_{1} & A_{2} & 0 & 0 & A_{3} & A_{4} \\
0 & 0 & 0 & 0 & 0 & 1 & 0 & 0 & 0 & 0 & 0 & 0 & 0 & 0 & 0 & 0 \\
0 & 0 & 0 & 0 & 0 & 0 & 1 & 0 & 0 & 0 & 0 & 0 & 0 & 0 & 0 & 0 \\
0 & 0 & 0 & 0 & 0 & 0 & 0 & 0 & 0 & 1 & 0 & 0 & 0 & 0 & 0 & 0 \\
0 & 0 & 0 & 0 & 0 & 0 & 0 & 0 & 0 & 0 & 1 & 0 & 0 & 0 & 0 & 0 \\
0 & 0 & 0 & 0 & 0 & 0 & 0 & 0 & 0 & 0 & 0 & 0 & 0 & 0 & 0 & 0
\end{array}\right]
$$




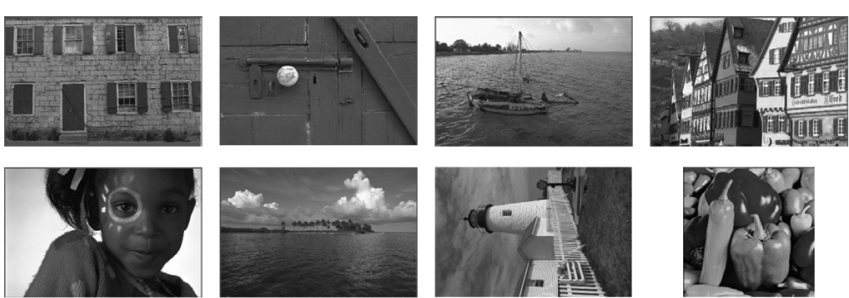

Fig. 12. Eight test images: (left to right and top to bottom) Wall, Door, Boat, House, Girl, Sky, Lighthouse, and Pepper.

TABLE I

PSNR (DB) OF EIGHT TEST IMAGES USING DIFFERENT ALGORITHMS

\begin{tabular}{|c|c|c|c|c|c|c|}
\hline Images & LMMSE [2] & NEDI [3] & Bicubic [17] & SAI [1] & LLR [12] & Proposed \\
\hline Wall & 25.069 & 24.827 & 25.208 & 25.223 & 25.172 & $\mathbf{2 5 . 4 2 5}$ \\
\hline Door & 33.113 & 33.042 & 33.069 & 33.327 & 33.422 & $\mathbf{3 3 . 5 4 4}$ \\
\hline Boat & 26.588 & 26.597 & 26.593 & 26.788 & 26.682 & $\mathbf{2 6 . 9 7 0}$ \\
\hline House & 22.45 & 22.074 & 22.403 & 22.51 & 22.175 & $\mathbf{2 2 . 6 9 4}$ \\
\hline Girl & 32.719 & 32.751 & 32.654 & 33.116 & 33.196 & $\mathbf{3 3 . 3 0 6}$ \\
\hline Sky & 30.106 & 30.055 & 30.073 & 30.166 & 30.188 & $\mathbf{3 0 . 3 6 6}$ \\
\hline Lighthouse & 27.024 & 26.088 & 27.001 & 26.727 & 26.911 & $\mathbf{2 7 . 3 7 6}$ \\
\hline Pepper & 33.202 & 33.014 & 32.848 & 33.533 & 33.728 & $\mathbf{3 3 . 7 7 5}$ \\
\hline Average & 28.783 & 28.556 & 28.731 & 28.923 & 28.934 & $\mathbf{2 9 . 1 8 2}$ \\
\hline
\end{tabular}

where only the center pixel (the fifth element in $\boldsymbol{X}$ ) is replaced by the estimated value. The local window is shifted in raster scan to estimate the next pixel by using the updated parameters $\boldsymbol{A}, \boldsymbol{B}$, and $\boldsymbol{W}$, and (21). After interpolating the center pixels, the adjacent pixels (e.g., the missing HR pixel on top of $X_{c}$ in Fig. 6) can be interpolated by the same steps using the scaled and rotated (by $45^{\circ}$ ) local windows, where the interpolated center pixels are used as "LR pixels."

\section{EXPERIMENTAL RESULTS}

Our experimental work was done on an Intel-i7, $3 \mathrm{GHz}$ computer system (single thread was used). Eight natural images (seven from Kodak) as shown in Fig. 12 were used for testing the robustness and accuracy of the proposed method in different situations. The image sizes range from $512 \times 512$ to $768 \times 512$. The images were downsampled without low-pass filtering and upsampled two times. We implemented the proposed method using (21), the local linear regression (LLR) [12], and bicubic convolution interpolator [17], whereas the execution codes of NEDI [3], linear minimum mean square error (LMMSE) [2], and SAI [1] were obtained from the authors' corresponding websites, respectively. The technique of regularization of filter coefficient from [12] has also been applied to our approach; however, this only accounts for a very slight further improvement in PSNR (about $0.007 \mathrm{~dB}$ ), as compared with those without using it in our approach.

As shown in Tables I-III, the proposed method, which applies the WLS estimation to SAI, gives the highest PSNR (in decibels), SSIM [18], and FSIM [19] on average. The average PSNR (in decibels) of the proposed method is $0.248,0.259,0.451$, 0.626 , and $0.399 \mathrm{~dB}$ higher than that of the LLR, SAI, bicubic, NEDI, and LMMSE, respectively. Note that SAI performs exceptionally well in image Pepper which involves a large portion of consistent geometric structures across resolutions, and performs relatively bad in image Lighthouse which involves severe mismatch issue of "geometric duality" In almost all test images,
TABLE II

SSIM [18] OF EIght TeSt IMAges Using DifFERENT ALgorithMS

\begin{tabular}{|c|c|c|c|c|c|c|}
\hline Images & LMMSE [2] & NEDI [3] & Bicubic [17] & SAI [1] & LLR [12] & Proposed \\
\hline Wall & 0.7560 & 0.7393 & 0.7659 & 0.7647 & 0.7621 & $\mathbf{0 . 7 6 9 2}$ \\
\hline Door & 0.8826 & 0.8802 & 0.8842 & 0.8867 & 0.8881 & $\mathbf{0 . 8 9 1 0}$ \\
\hline Boat & 0.8006 & 0.7990 & 0.8041 & 0.8085 & 0.8086 & $\mathbf{0 . 8 1 4 1}$ \\
\hline House & 0.7782 & 0.7572 & 0.7771 & 0.7804 & 0.7750 & $\mathbf{0 . 7 8 4 4}$ \\
\hline Girl & 0.9062 & 0.9078 & 0.9088 & 0.9117 & 0.9133 & $\mathbf{0 . 9 1 5 1}$ \\
\hline Sky & 0.8407 & 0.8394 & 0.8426 & 0.8441 & 0.8449 & $\mathbf{0 . 8 4 8 6}$ \\
\hline Lighthouse & 0.8426 & 0.8378 & 0.8454 & 0.8465 & 0.8481 & $\mathbf{0 . 8 5 2 2}$ \\
\hline Pepper & 0.8802 & 0.8822 & 0.8759 & 0.8824 & $\mathbf{0 . 8 8 7 6}$ & 0.8873 \\
\hline Average & 0.8358 & 0.8303 & 0.8380 & 0.8406 & 0.8409 & $\mathbf{0 . 8 4 5 2}$ \\
\hline
\end{tabular}

TABLE III

The FSIM [19] OF EIght TeSt IMAges Using DifFERENT ALgorithmS

\begin{tabular}{|c|c|c|c|c|c|c|}
\hline Images & LMMSE [2] & NEDI [3] & Bicubic [17] & SAI [1] & LLR [12] & Proposed \\
\hline Wall & 0.9387 & 0.9307 & 0.9404 & 0.9409 & 0.9406 & $\mathbf{0 . 9 4 3 0}$ \\
\hline Door & 0.9592 & 0.9592 & 0.9607 & 0.9604 & 0.9676 & $\mathbf{0 . 9 6 8 5}$ \\
\hline Boat & 0.9449 & 0.9401 & 0.9457 & 0.9471 & 0.9457 & $\mathbf{0 . 9 4 8 4}$ \\
\hline House & 0.9395 & 0.9325 & 0.9382 & 0.9396 & 0.9394 & $\mathbf{0 . 9 4 2 5}$ \\
\hline Girl & 0.9741 & 0.9761 & 0.9753 & 0.9757 & 0.9784 & $\mathbf{0 . 9 7 8 9}$ \\
\hline Sky & 0.9474 & 0.9496 & 0.9483 & 0.9478 & 0.9541 & $\mathbf{0 . 9 5 5 7}$ \\
\hline Lighthouse & 0.9542 & 0.9506 & 0.9507 & 0.9545 & 0.9527 & $\mathbf{0 . 9 5 4 5}$ \\
\hline Pepper & 0.9809 & 0.9801 & 0.9774 & 0.9817 & 0.9829 & $\mathbf{0 . 9 8 3 1}$ \\
\hline Average & 0.9548 & 0.9523 & 0.9545 & 0.9559 & 0.9576 & $\mathbf{0 . 9 5 9 3}$ \\
\hline
\end{tabular}

the proposed method gives the highest PSNR (dB), SSIM and FSIM.

Fig. 13 shows a portion of the Lighthouse image. It is obvious that the NEDI and SAI suffer from interpolation artifacts around the fence. Although this paper of SAI suggested using a cost function to detect overfitting, this method does not essentially solve the mismatch issue of "geometric duality" and a large threshold would eliminate the chance of interpolating "usable" pixels. The proposed method also applies a very gentle cost function method (which contributes to $0.02 \mathrm{~dB}$ overall, and $0.1 \mathrm{~dB}$ improvement in PSNR in the Lighthouse image) to reject extreme cases of overfitting. It is observable that the edges pointed by the red arrows are aliased areas resulting from LMMSE and bicubic interpolation.

Fig. 14 shows a portion of the Pepper image. Again, NEDI, SAI and the proposed method produce some visually similar edges pointed by the red arrows. However, the PSNR (dB) and SSIM of the proposed method are higher than that of the other methods. This suggests its fidelity issue among other methods. One can examine the image produced by NEDI in details and find that small edges (well below the red arrow) are blurred and there are directional artifacts near the small edges. This kind of nonlocal effect is due to nonadaptive weightings during the parameter estimation. The SAI method uses a comparably small local window such that the nonlocal effects are alleviated. However, due to the nonrobustness of the least-squares estimation, the SAI is sensitive to noise and outliner, resulting in lower fidelity images, compared to the proposed method, as justified in Table I. This kind of sensitivity is visually observable by looking at the directional artifacts in the smooth region. The SAI produces some slightly more visible directional lines in the smooth region than the proposed method. For the other two methods, LMMSE produces the second worst edge, and bicubic produces severely aliased edges due to nonadaptive filtering. Fig. 15 shows portions of the images Wall and 


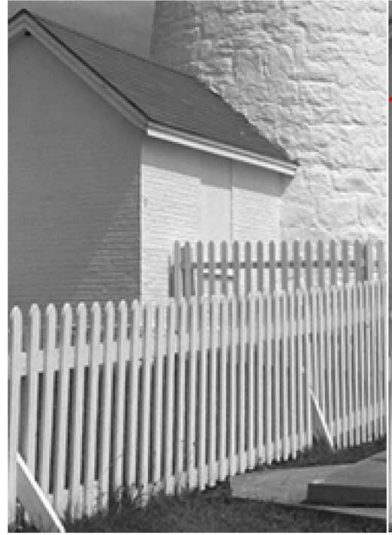

(a)

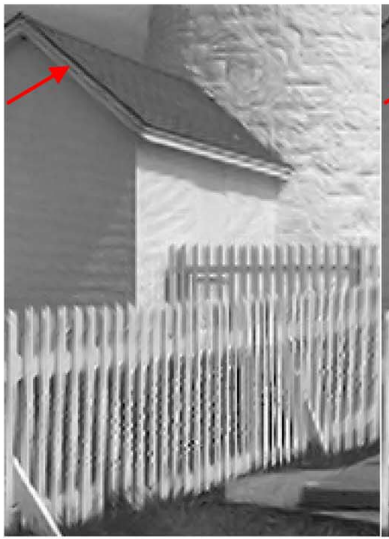

(c)

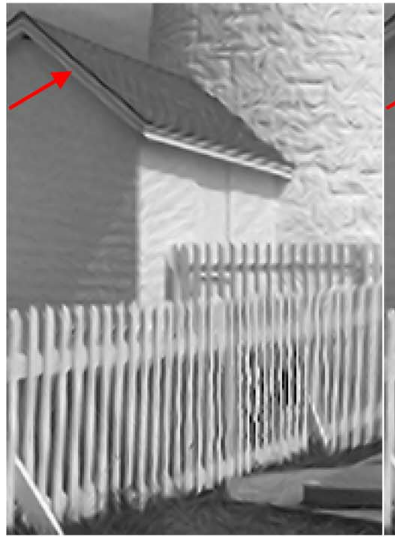

(e)

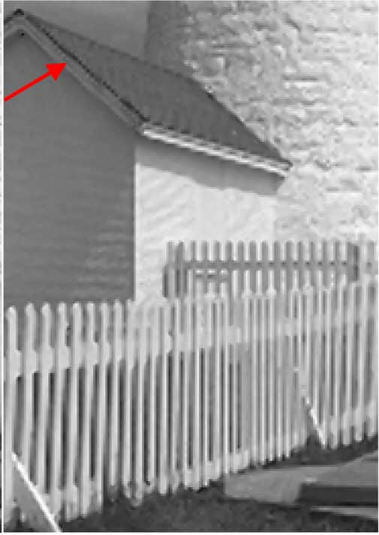

(b)

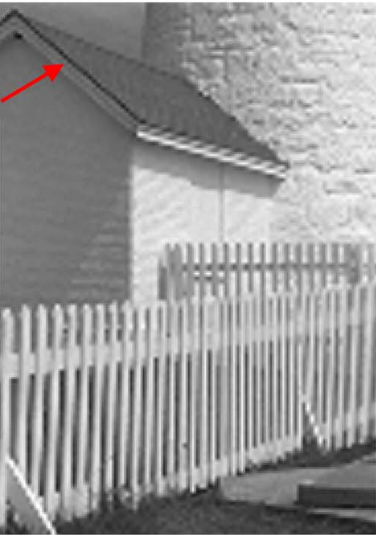

(d)

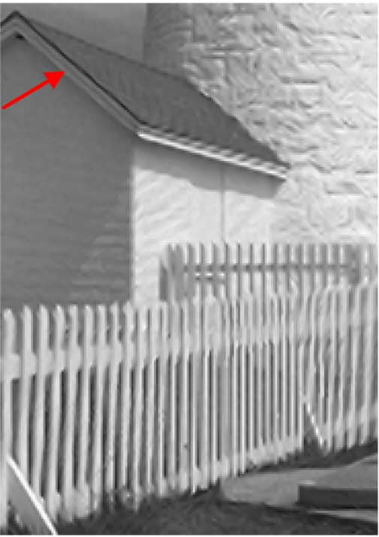

(f)
Fig. 13. Portions of image Lighthouse. From left to right and top to bottom, they are (a) the original HR image and interpolated images using (b) LMMSE [2], (c) NEDI [3], (d) Bicubic [17], (e) SAI [1], and the (f) proposed method.

Door. These portions also show that the proposed method has advantages in image structures with small details, compared to SAI.

\section{CONCLUSION}

In this paper, we have presented a robust soft-decision interpolation algorithm using WLS estimation for both parameter and data estimation steps. For parameter estimation, the weights are modeled by the geometric similarity to address the mismatch issues of "geometric duality." For data estimation, the

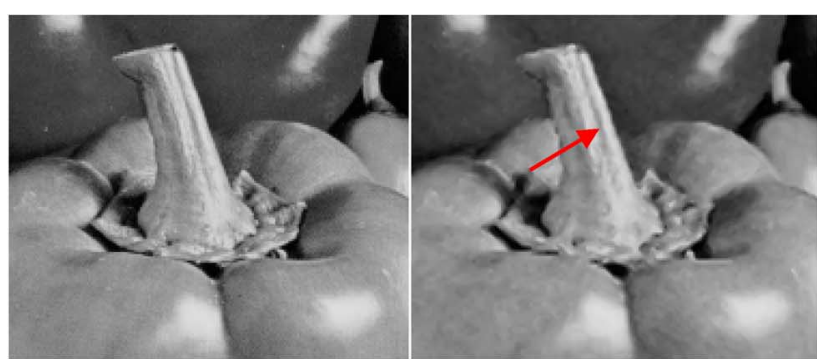

(a)

(b)

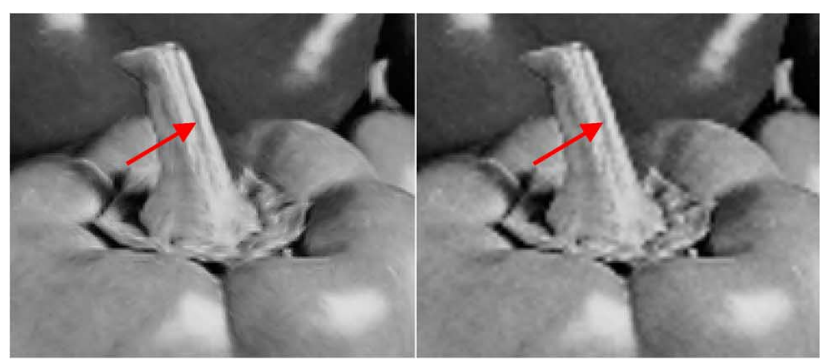

(c)

(d)

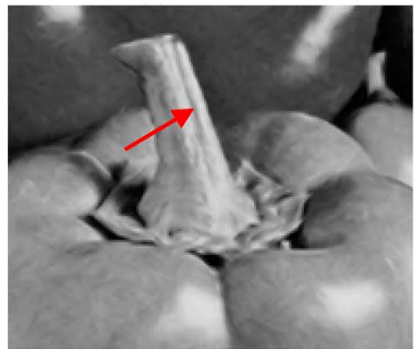

(e)

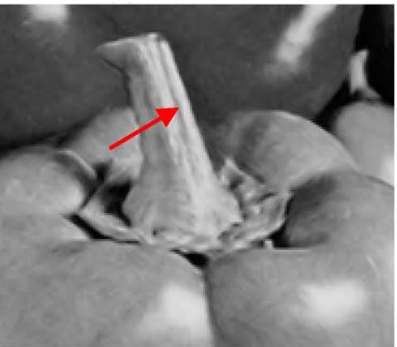

(f)

Fig. 14. Portions of image Pepper. From left to right and top to bottom, they are (a) the original HR image and interpolated images using (b) LMMSE [2], (c) NEDI [3], (d) Bicubic [17], (e) SAI [1], and (f) the proposed method.

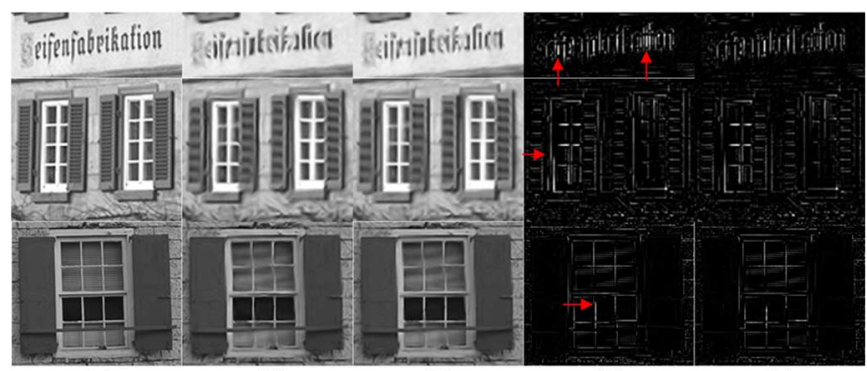

(a)

(b)

(c)

(d)

(e)

Fig. 15. Portions of image Wall and House. (a) Original HR image and interpolated images using (b) SAI [1], and (c) the proposed method and their absolute errors multiplied by two in (d) and (e).

weights are modeled by the well-known bilateral filter. Experimental results have shown that the proposed method has better performance, compared with the original SAI method and some other sophisticated methods in the literature in terms of objective and subjective evaluation. The proposed WLS estimation involves an additional two to three times computational cost, compared with that of the SAI, due to the brute-force implementation of the bilateral filter, the proposed geometric similarity metric, and the additional matrix operations. An enhancement work is to reduce the computational burden by optimizing further the whole process, particularly the widely available fast 
approaches for the bilateral filter and the iterative methods, instead of closed-form solutions, which require computationally demanding matrix inverses.

Alternatively, we may also reduce the computational burden by sacrificing some quality of the proposed algorithm. For the parameter estimation, we may replace the WLS estimation by the intuitive bilateral filter. For the soft-decision estimation, we can use the fixed-point strategy to avoid the high-dimensional matrix inverse. Our initial experimental results show that the computational cost is much lower while there could be a degradation of $0.1 \mathrm{~dB}$ PSNR. This is a fruitful direction for further research.

\section{REFERENCES}

[1] X. Zhang and X. Wu, "Image interpolation by adaptive 2-D autoregressive modeling and soft-decision estimation," IEEE Trans. Image Process., vol. 17, no. 6, pp. 887-896, Jun. 2008.

[2] L. Zhang and $\mathrm{X}$. Wu, "An edge-guided image interpolation algorithm via directional filtering and data fusion," IEEE Trans. Image Process., vol. 15, no. 8, pp. 2226-2238, Aug. 2006.

[3] X. Li and M. T. Orchard, "New edge-directed interpolation," IEEE Trans. Image Process., vol. 10, no. 10, pp. 1521-1527, Oct. 2001.

[4] K. Jensen and D. Anastassiou, "Subpixel edge localization and the interpolation of still images," IEEE Trans. Image Process., vol. 4, no. 3, pp. 285-295, Mar. 1995.

[5] W. Dong, L. Zhang, G. Shi, and X. Wu, "Image deblurring and superresolution by adaptive sparse domain selection and adaptive regularization," IEEE Trans. Image Process., vol. 20, no. 7, pp. 1838-1857, Jul. 2011.

[6] W. Dong, L. Zhang, G. Shi, and X. Wu, "Nonlocal back-projection for adaptive image enlargement," in Proc. IEEE Int. Conf. Image Process., Cairo, Egypt, Nov. 7-10, 2009, pp. 349-352.

[7] G. Shi, W. Dong, X. Wu, and L. Zhang, "Context-based adaptive image resolution upconversion," J. Electron. Imag., vol. 19, no. 1, p. 013 008, Jan.-Mar. 2010.

[8] S. Farsiu, M. D. Robinson, M. Elad, and P. Milanfar, "Fast and robust multi-frame super-resolution," IEEE Trans. Image Process., vol. 13, no. 10, pp. 1327-1344, Oct. 2004.

[9] P. J. Huber, Robust Statistics. New York: Wiley, 1981.

[10] R. Xiong, W. Ding, S. Ma, and W. Gao, "A practical algorithm for tanner graph based image interpolation," in Proc. IEEE 17th Int. Conf. Image Process., , Hong Kong, Sep. 2010, pp. 1989-1992.

[11] X. Zhang, S. Ma, Y. Zhang, L. Zhang, and W. Gao, "Nonlocal edge-directed interpolation," in Proc. 10th Pacific Rim Conf. Multimedia-Advances in Multimedia Information Processing, 2009, vol. 5879, Lecture Notes in Computer Science, pp. 1197-1207.

[12] X. Liu, D. Zhao, R. Xiong, S. Ma, and W. Gao, "Image interpolation via regularized local linear regression," in Proc. PCS, Dec. 8-10, 2010, pp. 118-121.

[13] K. W. Hung and W. C. Siu, "Improved image interpolation using bilateral filter for weighted least square estimation," in Proc. IEEE Int. Conf. Image Process., , Hong Kong, Sep. 26-29, 2010, pp. 3297-3300.

[14] C. Tomasi and R. Manduchi, "Bilateral filtering for gray and color images," in Proc. 6th IEEE Int. Conf. Comput. Vis., Bombay, India, Jan. 1998 , pp. 836-846.

[15] H. Takeda, S. Farsiu, and P. Milanfar, "Kernel regression for image processing and reconstruction," IEEE Trans. Image Process., vol. 16, no. 2, pp. 349-366, Feb. 2007.

[16] Official SAI program [Online]. Available: http://www.ece.mcmaster.ca/ xwu/

[17] R. G. Keys, "Cubic convolution interpolation for digital image processing," IEEE Trans. Acoust., Speech, Signal Process., vol. ASSP-29, no. 6, pp. 1153-1160, Dec. 1981.

[18] Z. Wang, A. C. Bovik, H. R. Sheikh, and E. P. Simoncelli, "Image quality assessment: From error visibility to structural similarity," IEEE Trans. Image Process., vol. 13, no. 4, pp. 600-612, Apr. 2004.

[19] L. Zhang, L. Zhang, X. Mou, and D. Zhang, "FSIM: A feature similarity index for image quality assessment," IEEE Trans. Image Process., vol. 20, no. 8, pp. 2378-2386, Aug. 2011.

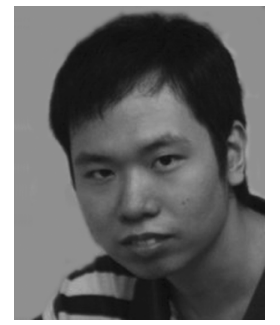

Kwok-Wai Hung (S'09) received the B.Eng. (Hons) degree in electronic and information engineering, in 2009, from The Hong Kong Polytechnic University, Hong Kong, where he is currently working toward the Ph.D. degree under the supervision of Prof. W.C. Siu.

His research interests include image and video signal processing, and image and video interpolation and superresolution.

Mr. Hung was the recipient of several awards and scholarships during his undergraduate study, including Dean's Honors List, Technical Excellent Award of his final-year project, and postentry scholarship for current students on UGC-funded undergraduate programs.

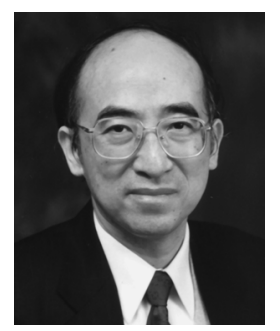

Wan-Chi Siu (S'77-M'77-SM'90-F'12) received the M.Phil. degree from The Chinese University of Hong Kong, Hong Kong, in 1977, and the $\mathrm{Ph} . \mathrm{D}$. degree from the Imperial College of Science, Technology and Medicine, University of London, London, U.K., in 1984

He joined The Hong Kong Polytechnic University, Hong Kong, as a Lecturer in 1980 and has been Chair Professor in the Department of Electronic and Information Engineering (EIE) since 1992. He was Head of EIE and subsequently Dean of the Engineering Faculty between 1994 and 2002. Prof. Siu is currently the Director of the Centre for Signal Processing of the same university. He is an expert in digital signal processing, specializing in fast algorithms and video coding, and has published 380 research papers, more than 160 of which appeared in international journals, such as the IEEE TRANSACTIONS ON IMAGE PROCESSING. He is a coeditor of the book Multimedia Information Retrieval and Management (Springer, 2003). His work on fast computational algorithms (such as DCT) and motion estimation algorithms has been well received by academic peers, with good citation records, and a number of which are now being used in hi-tech industrial applications, such as modern video surveillance and video codec design for high-definition television systems of some million dollar contract consultancy works. His research interests include transforms, image coding, transcoding, 3-D videos, wavelets, and computational aspects of pattern recognition.

Prof. Siu is the Vice President-Conference (Elect) of the IEEE Signal Processing Society (2012-2014). He is currently an Associate Editor for the IEEE TRANSACTIONS ON IMAGE PROCESSING and a member of the editorial board of a number of other journals such as Journal of VLSI Signal Processing Systems for Signal, Image, Video Technology. He was a Guest Editor and an Associate Editor for the IEEE TRANSACTIONS ON CIRCUITS AND SYSTEMS. He is a very popular lecturing staff member within the University, whereas outside the University, he has been a keynote speaker of more than ten international/national conferences in the recent ten years and an invited speaker of numerous professional events, such as the IEEE CPM 2002 (keynote speaker, Taipei, Taiwan); IEEE Intelligent Multimedia, Video and Speech Processing 2004 (keynote speaker, Hong Kong); IEEE International Conference on Information, Communications \& Signal Processing 2007 (invited speaker, Singapore); and IEEE International Conference on Acoustics, Speech, and Signal Processing 2008 (keynote speaker, Zhenjiang). He is the organizer of many international conferences, including the MMSP'08 (Australia) as General Co-Chair, and three IEEE Society sponsored flagship conferences: ISCAS'1997 as Technical Program Chair; ICASSP'2003 as the General Chair; and recently ICIP'2010 as the General Chair (2010 IEEE International Conference on Image Processing, which was held in Hong Kong, 26-29 September 2010). He is a member (2010-2012) of the Engineering Panel and also was a member of the Physical Sciences and Engineering Panel (1991-1995) of the Research Grant Council, Hong Kong Government. In 1993-1994, he chaired the first Engineering and Information Technology Panel of the Research Assessment Exercise to assess the research quality of 19 departments from all universities in Hong Kong and initiated to set up a set of objective indicators to assess the basic research quality of academia, which gives substantial impact to the research culture in Hong Kong for the recent 18 years. He is also the recipient of a number of awards, including the Distinguished Presenter Award (1997, HK), IEEE Third Millennium Medal (2000, USA), the Best Teacher Award (2003, HK), the Outstanding Award in Research (2003, HK), Plaque for Exceptional Leadership (2003, IEEE SPCB, USA), and Honorable Mention Winner Award (Pattern Recognition, 2004, USA). 